This is the author's accepted manuscript of the article published in Nature Immunology. The final authenticated version is available online at: https://doi.org/10.1038/s41590-021-00924-2

\title{
Lymphoid stromal cells proGrem dendritic cell homeostasis
}

Antonio P. Baptista ${ }^{1,2}$, Michael Y. Gerner ${ }^{3}$

1 Laboratory of Immunoregulation and Mucosal Immunology, VIB-UGhent Center for Inflammation Research, Ghent, Belgium

${ }^{2}$ Department of Internal Medicine and Pediatrics, Ghent University, Ghent, Belgium

${ }^{3}$ Department of Immunology, University of Washington School of Medicine, Seattle, WA

Correspondence: antonio.baptista@irc.vib-ugent.be; gernermy@uw.edu

\section{Single-cell technologies reveal the building blocks of secondary lymphoid organs, identifying Grem1 ${ }^{+}$fibroblastic reticular cells (FRCs) as critical niche cells for resident dendritic cell homeostasis and T cell immunity.}

Every well-designed house starts with a good architectural plan, a solid foundation, and strong structural framing. Together these elements demarcate the organization of all other interior components, and in the long run provide us with shelter, influence how we interact with one another, and shape how we live our lives. Similarly, the architectural scaffold of secondary lymphoid organs (SLOs), composed of diverse subpopulations of stromal cells, dictates the spatial arrangement of different innate and adaptive immune cells, provides factors essential for their survival, and plays a key role in the timely and efficient generation of immune defense ${ }^{1,2}$. In this issue of Nature Immunology, Kapoor et al. use single-cell RNA sequencing (scRNA-seq) approaches to shed further light on the foundational building blocks of SLOs, providing evidence for the existence of a spatially constrained fibroblastic reticular cell (FRC) subset in lymph nodes (LNs), spleen and Peyer's patches, involved in the local homeostatic maintenance of dendritic cells (DCs), and in promoting the generation of adaptive immunity ${ }^{3}$ (Fig. 1).

SLOs promote host defense by concentrating in one location DCs displaying information about the state of tissues, and recirculating lymphocytes in search of their cognate antigens. Appropriate localization of these innate and adaptive cells is essential for efficient cell-cell crosstalk and timely generation of responses. Recent work using high-dimensional imaging tools has revealed complex spatial patterns of distinct DC and T cell subsets and the existence of discrete immune microenvironments in SLOs ${ }^{4,6}$. Generation of these microenvironments is thought to be driven by chemotactic and adhesion cues provided by the underlying stromal cell network. This same stromal network not only supplies critical survival cues to different immune cells (e.g. interleukin 7 for $T$ cells, delta-like ligand 1 for $D C s^{1,2}$ ) but can also directly influence immune responses during inflammation (e.g. major histocompatibility complex II (MHCII) expression, Nitric oxide, etc. ${ }^{1,7,8}$ ). While originally described as a relatively simple collection of stromal cells, composed of $\mathrm{T}$ cell zone-associated fibroblastic reticular cells (FRCs), B cell follicle-associated follicular dendritic cells (FDCs), subcapsular sinus-associated marginal reticular cells (MRCs), as well as blood and lymphatic endothelial cells, recent studies using 
scRNA-seq have begun to unravel marked heterogeneity in these populations ${ }^{2,9,10}$. The current study by Kappor et al. validates these past efforts, while further extending them by defining a novel FRC subset involved in DC maintenance, as well as for the first time, providing a glimpse into the complexity of stromal cells in human $\mathrm{LNs}^{3}$.

Specifically, the authors identified several new non-endothelial FRC subsets, and in particular, a subpopulation of FRCs expressing the bone morphogenetic protein antagonist Gremlin1 $(G r e m 1)^{2}$. They next used the Grem1 promoter region to generate tamoxifen-inducible yellow fluorescent protein reporter (Grem1-YFP) and diphtheria receptor depleter (Grem1-YFP/iDTR) mouse strains, which together allowed them to characterize the location and function of the newly identified stromal cell type in vivo. They found that Grem $1^{+}$FRCs were predominantly localized in the peripheral paracortex of SLOs, or more simply, at the T/B boundary in both LNs and spleen. Similar localization has been previously reported for cholesterol 25-hydroxylase (Ch25h)-expressing stromal cells involved in promoting B cell, T cell, and DC positioning and homeostasis $^{3,10,11}$. Indeed, Grem $1^{+}$FRCs were enriched for Ch25h mRNA expression, as well as expressed multiple chemotactic and adhesional modules implicated in cellular adhesion, recruitment, and homeostasis. Consistently, depletion of Grem1 ${ }^{+}$FRCs led to a severe reduction in DC cellularity and blunted $\mathrm{T}$ cell responses to immunization. This result suggests that Grem1 $1^{+}$FRCs are a cornerstone stromal cell type responsible for promoting DC homeostasis in SLOs and the generation of immunity.

In addition to their mouse studies, Kapoor et al. used scRNA-seq to characterize stromal cell heterogeneity in human $\mathrm{LNs}^{3}$. While observing somewhat reduced complexity in population diversity, the authors identified multiple key FRC populations with closely aligning molecular profiles to their mouse counterparts, including the newly described Grem $1^{+}$FRCs. While Grem $1^{+}$FRCs expressed similar chemotactic and adhesion factors in both species, certain factors were species-specific. Together, these findings support the notion that this cell subset is an important FRC population requiring further investigation and with likely translational relevance.

While revealing the molecular identity and some of the downstream functions of the Grem $1^{+}$ FRCs, much remains to be determined in future studies. In particular, the mechanisms of how Grem1 ${ }^{+}$FRCs exert their influence over DC homeostasis remain elusive. Depletion of these stromal cells led to reduced cellular turn-over, increased cell death, and subsequent loss of resident DC subsets in LNs. These changes were not associated with decreased pre-DC generation in the bone marrow nor the ability of pre-DCs to home to SLOs. The authors did find that Grem1 ${ }^{+}$FRCs were localized in relative proximity to both resident DC1 and DC2 populations, as well as pre-DCs after adoptive transfer. Computational analyses of receptorligand pairs predicted multiple possible mechanisms of intercellular communication between these cell types, although not all were conserved across species ${ }^{3}$. However, it remains unclear whether Grem1 ${ }^{+}$FRCs directly sustain resident DC homeostasis, or more simply provide a scaffolding niche to support this process. Consistent with the latter hypothesis, it should be noted that Grem1 ${ }^{+}$FRC ablation coincided with markedly reduced LN cellularity and that DCs are short-lived cells requiring continuous replenishing by bone marrow precursors ${ }^{12}$. As DCs are 
anchored directly in the FRC network ${ }^{1}$, loss of attachment to Grem1 ${ }^{+}$FRCs by itself could prevent completion of DC differentiation and lead to anoikis (cell-detachment-induced apoptosis). Both direct and indirect mechanisms are not mutually exclusive, with sensing of the local morphogenetic field (knowing where one is via communication events with the surrounding cellular and extracellular environment) being crucial to the adoption of proper cell state and adequate tissue patterning, and anoikis used to eliminate misplaced cells that would otherwise interfere with normal tissue function.

Intriguingly, while resident DC homeostasis was negatively affected by loss of Grem ${ }^{+} \mathrm{FRCs}$, the homeostasis of migratory DCs was not ${ }^{3}$. Given that the development of migratory DCs occurs in peripheral tissues, this finding further supports the notion of the predominant influence of $\mathrm{Grem} 1^{+}$FRCs in SLOs, as well as indicates that resident and migratory DC homeostasis is mediated by distinct mechanisms, which require future elucidation. In a similar vein, decreased adaptive immune responses observed in Grem1-FRC-depleted mice also support the dominant involvement of resident, not migratory, DCs in promoting $\mathrm{T}$ cell responses in subunit vaccination settings ${ }^{13,14}$.

The net effect of $\mathrm{Grem}^{+} \mathrm{FRC}$ depletion was impaired T cell responses ${ }^{3}$. While this effect may be secondary to reduced antigen-presentation by the fewer DCs present, a direct role of Grem $1^{+}$FRCs in sustaining $\mathrm{T}$ cell immunity cannot be ruled out. Indeed, FRCs have been shown to modulate effector $T$ cell function by both direct and indirect mechanisms ${ }^{1,7,8}$, as well as to stretch and proliferate to accommodate increased LN cellularity during immunological responses ${ }^{1}$. As DCs themselves are instrumental to the latter changes in FRCs, it could be that the loss of DCs after Grem $1^{+}$FRC depletion leads to global changes in the entire SLO stromal network. Whether such putative changes in the remaining stromal cell network help explain the additional reductions in overall $\mathrm{LN}$ mass and $\mathrm{CD}^{+}{ }^{+} \mathrm{T}$ cell cellularity observed in DT-treated animals requires further investigation. Grem $1^{+}$FRCs could also be directly involved in regulating cellular trafficking (entry and/or egress) through lymphoid organs as well as in $\mathrm{CD}^{+} \mathrm{T}^{\mathrm{T}}$ cell maintenance. Alternatively, given that $\mathrm{CD}^{+} \mathrm{T}$ cells require continuous $\mathrm{T}$ cell antigen receptor engagement for homeostatic maintenance, loss of $\mathrm{MHC}-\mathrm{II}$ expressing $\mathrm{DCs}$ alongside mislocalization of $\mathrm{CD}^{+} \mathrm{T}$ cells within the $\mathrm{T}$ zone ${ }^{4}$ may lead to impairments in $\mathrm{T}$ cell maintenance.

Single-cell omics technologies offer an unbiased, highly granular view into cellular heterogeneity, generating datasets that lead to many hypotheses and pressing questions. With the study by Kapoor et al. ${ }^{2}$, it becomes pressing to define the diverse and likely complex functional roles of the different stromal subpopulations in SLOs. Similarly, understanding how stromal cell biology changes in distinct inflammatory conditions, and how this influences different aspects of innate and adaptive responses (homeostasis, localization, cell-cell communication, etc.) will be of the essence. Moreover, it will be important to explore which of the identified cell clusters represent stable populations and which represent more transient cell states, as well as the molecular mechanisms regulating cellular transition(s). Resolving these questions will be aided by advanced computational approaches to deduce developmental trajectories and cell-cell communication pathways, as was done in the current study. As we 
continue to achieve a better understanding of stromal cell heterogeneity, it will be important to harmonize a common nomenclature for the different cell types, and across species, identified in different studies. Kapoor et al. have revealed an important subpopulation of FRCs involved in DC homeostasis and generation of immunity in SLOs ${ }^{2}$. In combination with other recent studies, one is left feeling that we both have a better understanding of stromal cell biology, and left marveling at the ever-increasing complexity of the structure-function relationships involved in host defense.

\section{Figure 1}

\section{Grem1 ${ }^{+}$FRCs build a niche for DC homeostasis and T cell immunity.}

Single-cell RNAseq identifies Grem $1^{+}$fibroblastic reticular cells (FRCs) among non-endothelial lymphoid stromal cells, which localize to the T/B border, where they sustain resident DC homeostasis and promote $\mathrm{T}$ cell responses.

\section{Acknowledgements}

A.P. Baptista is supported by a Marie-Sklodowska-Curie fellowship (EU project 898090). Research in the Gerner laboratory is supported by NIH grants R01Al134713, R21Al142667, R01Al1134246, 75N93019C00070, T32Al106677, T32GM007270; the National Science Foundation (NSF DGE-1762114), by the Washington Research Foundation postdoctoral fellowship, as well as by research funds from Roche Glycart AG.

\section{References}

1. Krishnamurty, A.T. \& Turley, S.J. Nat Immunol 21, 369-380 (2020).

2. Pikor, N.B., Cheng, H.W., Onder, L. \& Ludewig, B. J Immunol 206, 257-263 (2021)

3. Kapoor et al. Nat Immunol

4. Baptista, A.P. et al. Immunity 50, 1188-1201 e1186 (2019).

5. Qi, H., Kastenmuller, W. \& Germain, R.N. Annu Rev Cell Dev Biol 30, 141-167 (2014).

6. Stoltzfus, C.R. et al. Cell Rep 31, 107523 (2020).

7. Baptista, A.P. et al. Elife 3, e04433 (2014).

8. Lukacs-Kornek, V. et al. Nat Immunol 12, 1096-1104 (2011).

9. Pezoldt, J. et al. Nat Commun 9, 3903 (2018).

10. Rodda, L.B. et al. Immunity 48, 1014-1028 e1016 (2018).

11. Cyster, J.G., Dang, E.V., Reboldi, A. \& Yi, T. Nat Rev Immunol 14, 731-743 (2014)

12. Liu, K. et al. Nat Immunol 8, 578-583 (2007).

13. Gerner, M.Y., Torabi-Parizi, P. \& Germain, R.N. Immunity 42, 172-185 (2015).

14. Leal, J.M. et al. Sci Immunol 6, eabb9435 (2021). 
(1) Single cell SC heterogeneity

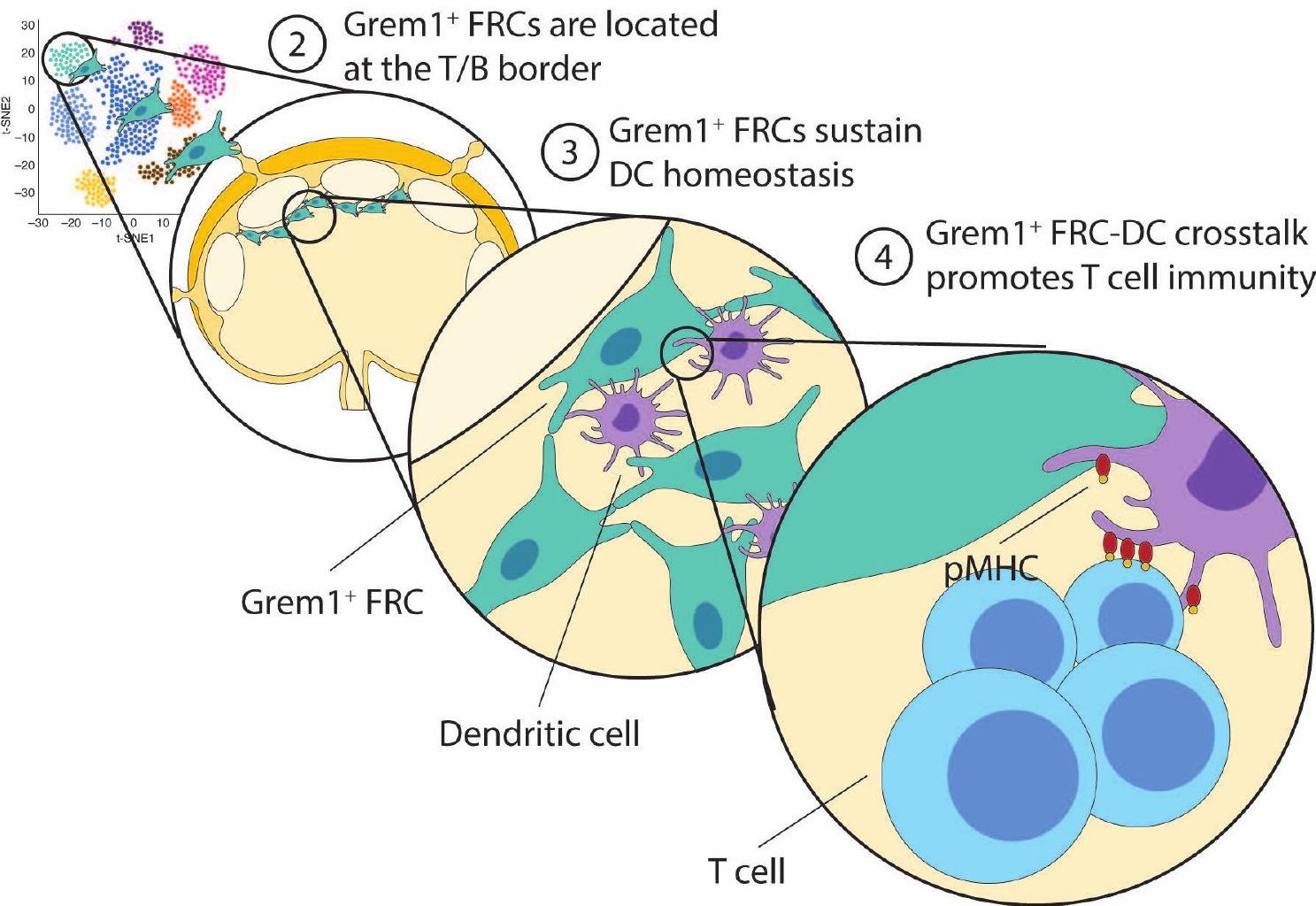

